\title{
Efeito do Tipo de Elemento de Rosca na Degradação de Polipropileno Durante Múltiplas Extrusões
}

\author{
Ana Clélia Babetto e Sebastião V. Canevarolo, \\ Departamento de Engenharia de Materiais, UFSCar
}

Resumo: A configuração da rosca de uma extrusora de dupla rosca modular pode ser projetada com vários tipos de elementos de condução e mistura, produzindo diferentes níveis de degradação no polímero fundido. Neste trabalho determinou-se o nível de degradação do polipropileno durante múltiplas extrusões em várias configurações de rosca, através de cromatografia por exclusão de tamanho e espectroscopia de infravermelho. Houve decréscimo do peso molecular e estreitamento da sua distribuição em função do número de extrusões e do aumento da agressividade da rosca, assim como, aumento da concentração de carbonilas e insaturações na cadeia polimérica. O elemento de mistura EM90 causou o maior nível de degradação e a adição de elementos de condução esquerdos ECE reduziram o nível de degradação.

Palavras-chave: Configuração de rosca, polipropileno, degradação, peso molecular.

\section{Effect of screw element on the polypropylene degradation during multiple extrusions}

Abstract: The screw configuration of a double screw extruder can be designed to contain kneading and conveying elements inducing different levels of degradation in the polymer melt. In this work the level of degradation in polypropylene have been measured after multiple extrusions for various screw configurations using Size Exclusion Chromatography and Infra-Red Spectroscopy. The average molecular weight and the polydispersity are reduced and the carbonyl and unsaturation indices increase as the number of extrusions and the agressiveness of the screw increase. The kneading element with 90 degrees caused the greater level of degradation and the addition of left hand elements have reduced the level of degradation.

Keywords: Screw configuration, polypropylene, degradation, molecular weight.

\section{Introdução}

Devido à grande importância comercial e industrial do polipropileno muito se tem pesquisado para melhorar o desempenho nos processos de sua síntese, composição e transformação. A extrusora de dupla rosca modular possui vantagens em relação à rosca simples em todas as etapas do processo de extrusão ${ }^{[1]}$. A configuração deste tipo de rosca pode incorporar vários tipos de elementos de condução (EC), mistura (EM) e de condução e mistura de passo esquerdo (ECE ou EME), produzindo diferentes níveis de cisalhamento no fundido. Os ECs, possuem vários fatores que influenciam na determinação do tipo de fluxo, tais como ângulo de condução, passo, largura e número de filetes por unidade de comprimento. Os EMs impõem ação cisalhante e de mistura no fundido. Existem vários tipos de EM, os mais comuns são formados por "discos", nos quais a largura e diferença angular entre eles, provocam níveis distintos de mistura, cisalhamento e transporte no material durante o fluxo ${ }^{[2-6]}$. Stade ${ }^{[7]}$ fez um estudo sobre tendências em projetos de roscas de extrusoras, constatando a importância da geometria dos elementos de mistura nas misturas distributiva e dispersiva. Os ECEs e EMEs, invertem o sentido do fluxo, forçando o material a retornar ao elemento anterior. Este tipo de elemento provoca a formação de uma barreira de fundido com grau de preenchimento $100 \%$ e, portanto, elevando a pressão nesta

Autor para correspondência: Sebastião V. Canevarolo, Universidade Federal de São Carlos, Departamento de Engenharia de Materiais, Via Washington Luiz, Km 235, C.P. 676, CEP:13565-905, São Carlos, SP, E-mail: caneva@power.ufscar.br 
região. Após o ECE ou EME o grau de preenchimento diminui, e, consequentemente, ocorre redução da pressão. A seleção da configuração de rosca deve ser otimizada com o intuito de atender às necessidades e características do processo. Efeitos de mistura, cisalhamento e transporte são vinculadas à sequência de elementos de rosca e portanto devem estar harmonicamente posicionados ao longo de todo o comprimento da rosca.

Durante o processo de extrusão, o material é exposto às condições de altas temperaturas e taxas de cisalhamento, as quais causam degradação, ocorrendo a combinação de degradação térmica, mecânica e química ${ }^{[8]}$. Tzoganakis et al. ${ }^{[9]}$ induziram a degradação do polipropileno por peróxido em uma extrusora de rosca simples. Variando a concentração de peróxidos, obtiveram amostras de polipropileno de vários pesos moleculares. Utilizando-se a cromatografia por exclusão de tamanho mediram o peso molecular médio e analisaram sua influência nas propriedades reológicas e mecânicas. Kim e White ${ }^{[10]}$ e Ebner e White ${ }^{[4]}$, analisaram o efeito da configuração de rosca no processamento através da degradação do polipropileno induzida por peróxidos. Hinsken et al., ${ }^{[11]} \mathrm{e}$ González-González et al. ${ }^{[12]}$ estudaram a degradação do polipropileno em múltiplas extrusões confirmando que sua degradação ocorre principalmente com cisão de cadeia, alterando o perfil da distribuição de peso molecular e reduzindo seu valor médio. A presença de grupos carbonílicos na cadeia foi determinada por espectroscopia no infravermelho.

O conhecimento da inter-relação entre o grau de cisalhamento imposto pela configuração da rosca e o nível de degradação é um importante instrumento para que sejam estabelecidas condições operacionais adequadas de processamento do polímero (taxa de alimentação, configuração e velocidade de rotação da rosca e perfil de temperatura). Neste trabalho analisou-se a degradação do polipropileno durante o processamento com várias configurações de roscas em múltiplas extrusões visando quantificar seu nível induzido por cada tipo de elemento de rosca. Para tanto empregou-se a cromatografia por exclusão de tamanho para acompanhar as mudanças nas curvas de distribuição de peso molecular e a espectroscopia no infravermelho para quantificar o aumento da concentração de carbonilas e insaturações na cadeia.

\section{Experimental}

\section{Extrusão}

Material: Polipropileno homopolímero (PP) H306, grau extrusão da OPP Petroquímica SA.

Equipamento: Extrusora dupla rosca modular corotativa totalmente interpenetrante ZSK 30 Werner \& Pfleiderer, com alimentador gravimétrico fabricado pela K-Tron.

\section{Metodologia:}

a) Configuração das roscas - O critério de projeto para a definição das configurações das roscas foi de analisar o efeito degradativo dos vários tipos de elementos de rosca. Construiu-se cinco configurações que estão esquematizadas na Figura 1, cujos elementos de rosca utilizados estão especificados na Tabela 1.

b) Condições de processo - Perfil de temperatura do barril dividido em seis zonas iniciando-se na alimentação: 210 - 230 - 240 - $250-240-235{ }^{\circ} \mathrm{C}$,

Tabela 1. Tipos de elementos de rosca utilizados.

\begin{tabular}{cc}
\hline Tipo de elemento de rosca & Especificação \\
\hline EA & Apoio, $10 \mathrm{~mm}$ de comprimento \\
EC1 & Condução direito, filete simples, $42 \mathrm{~mm}$ de passo e $42 \mathrm{~mm}$ de comprimento \\
EC2 & Condução direito, filete simples, $28 \mathrm{~mm}$ de passo e $28 \mathrm{~mm}$ de comprimento \\
EC3 & Condução direito, filete simples, $28 \mathrm{~mm}$ de passo e $14 \mathrm{~mm}$ de comprimento \\
EC4 & Condução direito, filete simples, $20 \mathrm{~mm}$ de passo e $20 \mathrm{~mm}$ de comprimento \\
ECE & Condução esquerdo, filete simples, $20 \mathrm{~mm}$ de passo e $10 \mathrm{~mm}$ de comprimento \\
EM45 & Mistura, 5 discos bi-lobulares, diferença angular $45^{\circ}$ e $28 \mathrm{~mm}$ de comprimento \\
EM90 & Idem anterior com diferença angular $90^{\circ}$ e $28 \mathrm{~mm}$ de comprimento \\
\hline
\end{tabular}


$\mathrm{CON}$

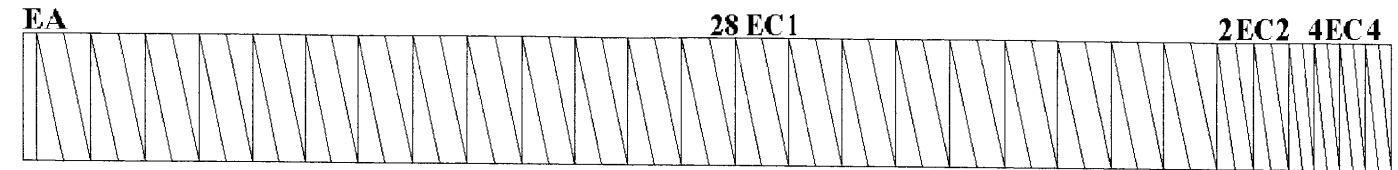

$\mathrm{C} 45$

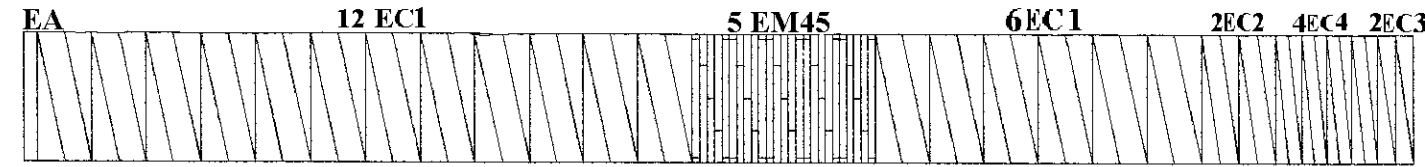

C45E

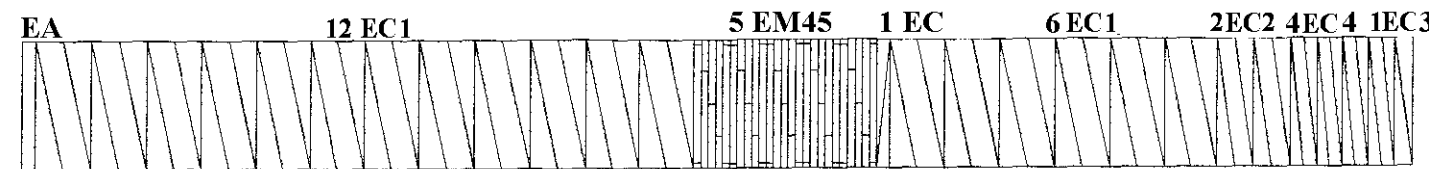

C90

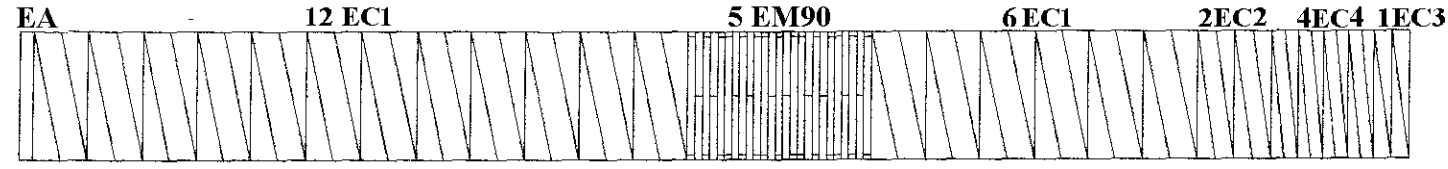

C90E

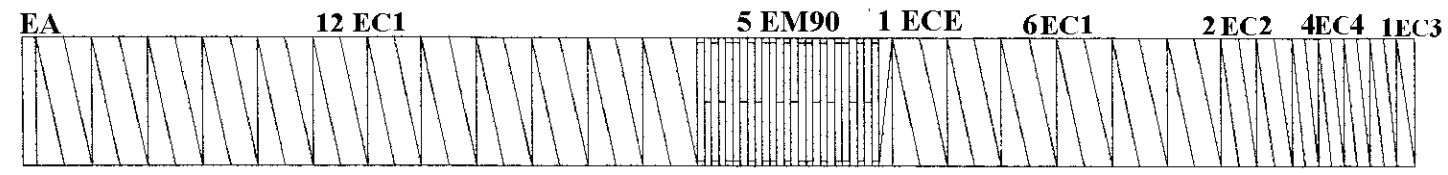

Figura 1. Configurações das roscas utilizadas

taxa de alimentação $5 \mathrm{Kg} / \mathrm{h}$ e velocidade de rotação da rosca de $100 \mathrm{rpm}$.

c) Metodologia de processamento - O polipropileno em grãos foi alimentado pelo alimentador gravimétrico previamente calibrado. Após extrusão e peletização, uma quantidade de amostra foi coletada e o restante realimentado na extrusora para reprocessamento, de forma a se obter amostras com múltiplas extrusões. Mediu-se o tempo de residência e sua distribuição online segundo Santos e Canevarolo ${ }^{[13,14]}$.

\section{Caracterização da degradação}

\section{Espectroscopia no Infravermelho}

A análise no infravermelho foi feita em filmes termoprensados em uma prensa Luxor, a $190^{\circ} \mathrm{C}$ e 10 $\mathrm{kg} / \mathrm{cm}^{2}$. Utilizou-se duas placas de latão previamente polidas e filmes de poliéster como anti-aderente. A espessura foi determinada por um micrômetro eletrônico variando numa faixa de $70-100 \mu \mathrm{m}$. Foram preparados vários filmes para cada amostra a fim de analisar a reprodutibilidade dos dados. Os filmes foram fixados em suportes de papel cartão para a análise de infravermelho, em equipamento Perkin-Elmer FTIR modelo Spectrum 1000. Obteve-se a linha de base ( dito "background") com 32 varreduras e $4 \mathrm{~cm}^{-1}$ de resolução na faixa de comprimento de onda de $4000-400 \mathrm{~cm}^{-1}$. Escolheu-se uma posição no filme que apresentasse espessura conveniente e constante, limitando-se a faixa de absorbância para um pico padrão. Os espectros foram obtidos nas mesmas condições de varredura da linha de base. Esta última era refeita a cada três análises.

\section{Cromatografia por exclusão de tamanho}

O cromatógrafo usado foi o Waters GPC $150 \mathrm{CV}$, com detector por índice de refração e três colunas Waters Styragel HT de tamanho médio de partícula de $10 \mu \mathrm{m}$, separando pesos moleculares na faixa de $10^{2}$ a $10^{6}$. As amostras foram solubilizadas à concentração de $0,1 \%$, em 1,2,4-triclorobenzeno (TCB) com anti-oxidante à $140^{\circ} \mathrm{C}$, filtradas e injetadas no equipamento a um fluxo de $1 \mathrm{ml} / \mathrm{min}$. Os cálculos foram feitos no programa Millennium GPC exportadas e re-apresentadas através do software Origin 5.0.

\section{Resultados e Discussões}

Degradação em função do número de extrusões

Espectros de FTIR foram obtidos para o polipropileno sem e com múltiplas extrusões em todas 
Babetto, A. C.; Canevarolo, S. V. - Degradação de Polipropileno durante múltiplas extrusões
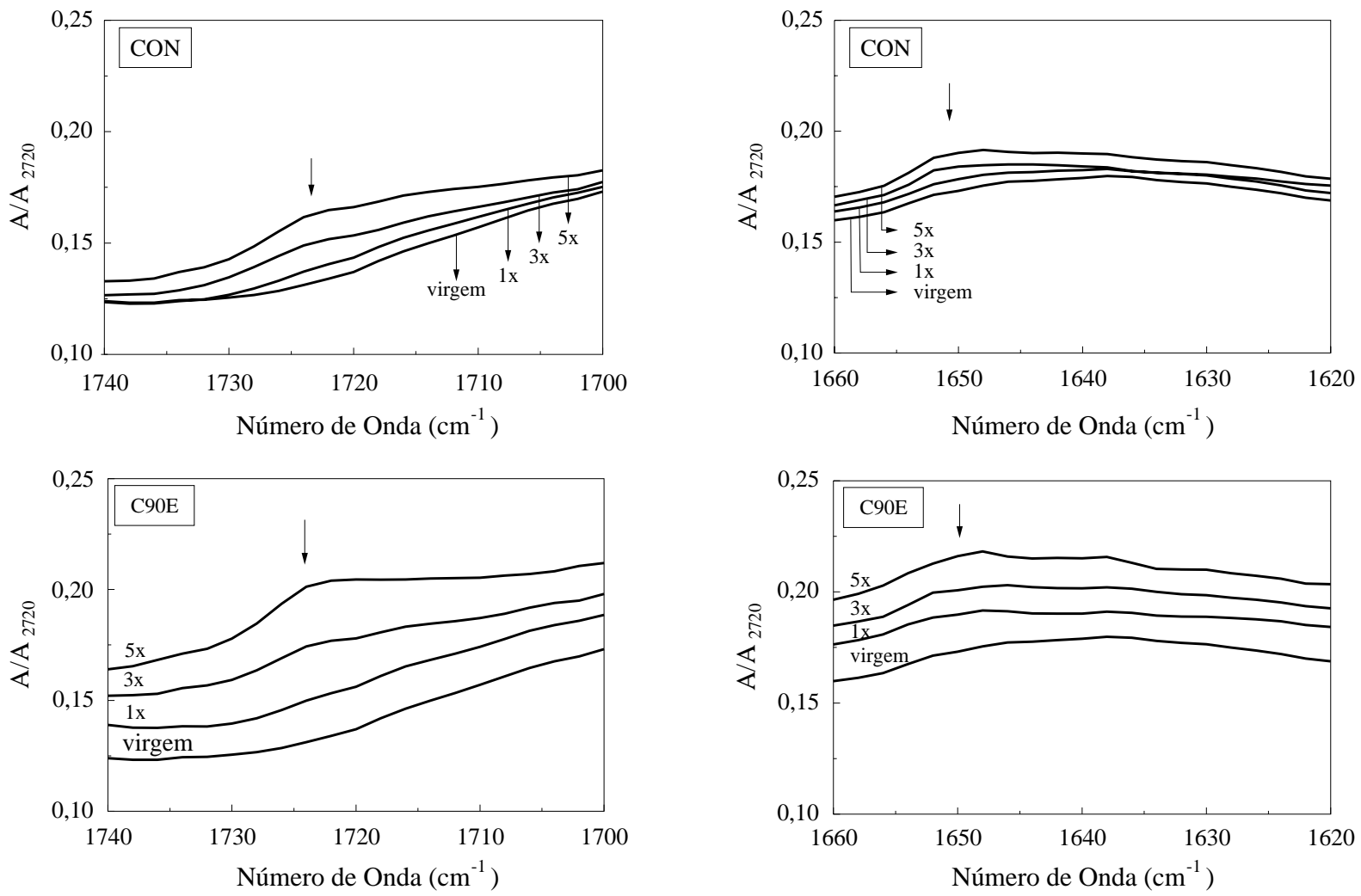

Figura 2. Aumento de intensidade de absorção no infravermelho em função do número de extrusões e da configuração da rosca.
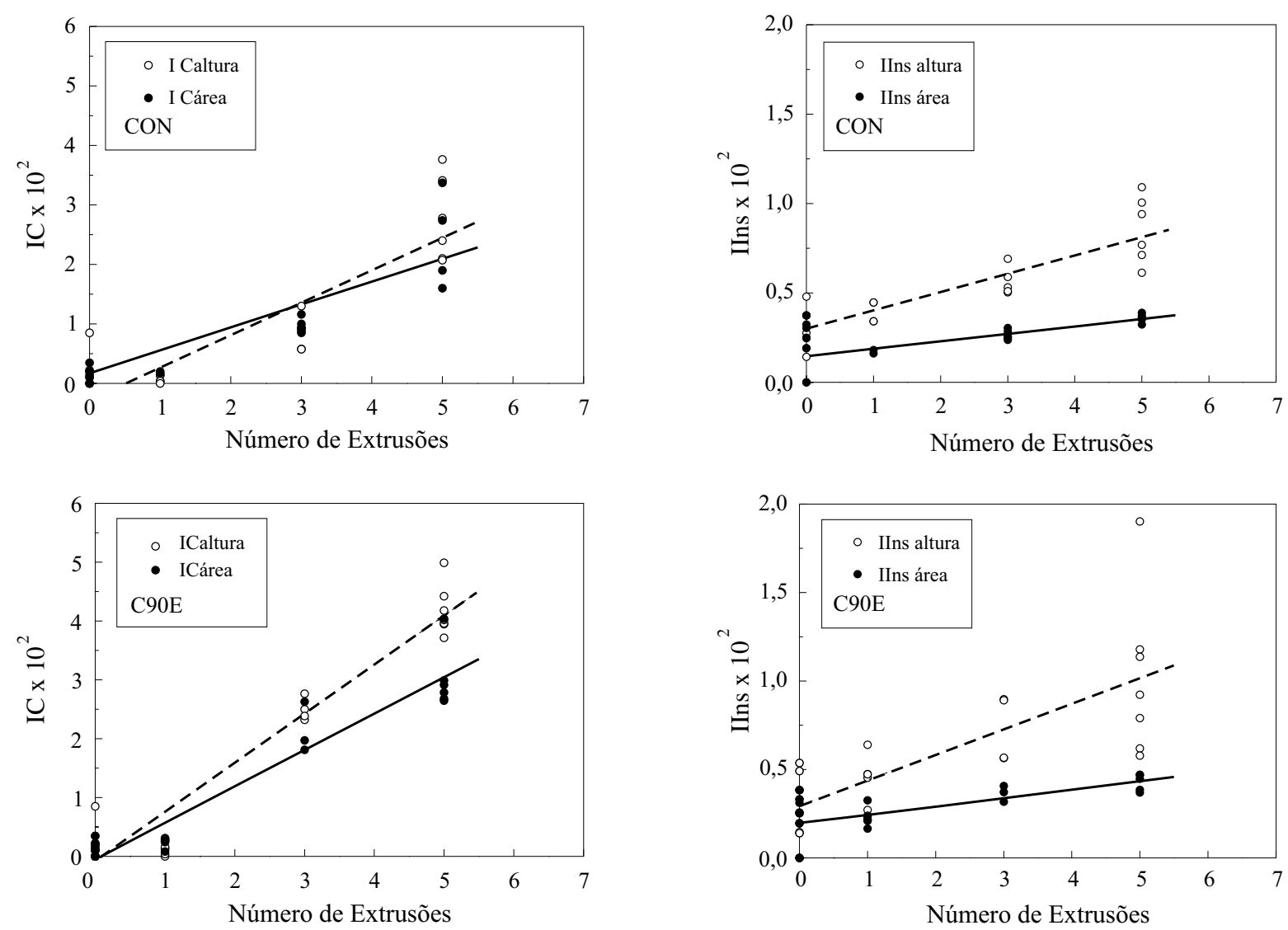

Figura 3. Índices de carbonila (IC) e de insaturação (IIns) em função do número de extrusões. 
as configurações de roscas estudadas. Para eliminar o efeito da não uniformidade das espessuras dos filmes, normalizou-se os espectros utilizando-se o pico a 2720 $\mathrm{cm}^{-1}$ como referência, relacionado às vibrações moleculares angular do grupo $\mathrm{CH}$ e axial do grupo $\mathrm{CH}_{3}$ como sugerido em outras referências ${ }^{[15,16,17]}$. O pico a $2720 \mathrm{~cm}^{-1}$, utilizado por Garton et alii ${ }^{[16]}$ para normalizar os espectros de IR, teve confirmação, através da comparação de absorções de outros picos, de que é insensível à degradação oxidativa do PP homopolímero e aos parâmetros morfológicos que o trabalho analisa. Na Figura 2 os espectros no infravermelho mostram o aumento da intensidade de absorção na região de absorção dos produtos da degradação oxidativa do $\mathrm{PP}$, indicando o progresso da degradação com o aumento do número de extrusões para as configurações de roscas $\mathrm{CON}$ e C90E. A presença de um ombro, à aproximadamente $1725 \mathrm{~cm}^{-1}$, confirma o aparecimento de grupos carbonílicos, e o pico na região próxima a $1650 \mathrm{~cm}^{-1}$, corresponde à presença de insaturações.

O índice de carbonila ${ }^{[16]}$ foi calculado a partir da razão entre as alturas ou áreas em unidades de absorbâncias do pico à $1725 \mathrm{~cm}^{-1} \mathrm{e}$ a do pico à 2720 $\mathrm{cm}^{-1}$. Da mesma forma calculou-se o Índice de
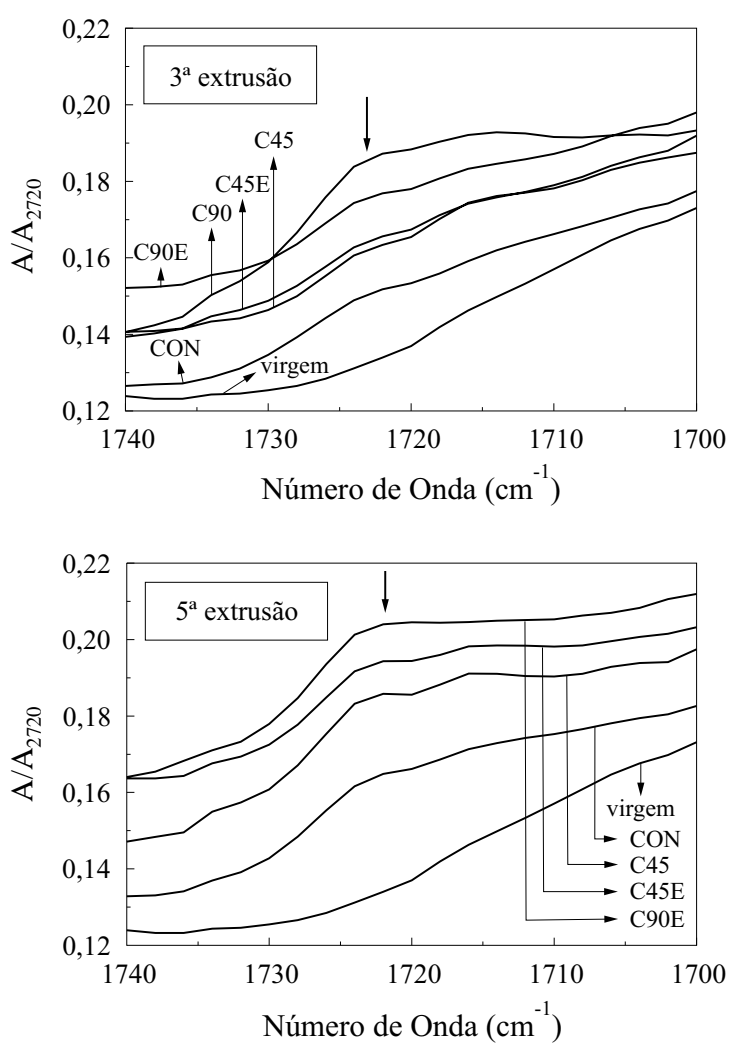

Insaturação (IIns), usando-se o pico correspondente à absorção das insaturações $\mathrm{C}=\mathrm{C}$ à $1650 \mathrm{~cm}^{-1}$. A Figura 3 apresenta o comportamento do IC e IIns em função do número de extrusões, para as configurações CON e C90E. Os gráficos mostram que tanto o índice de carbonila, como o índice de insaturação aumentam com o número de extrusões. $\mathrm{O}$ ajuste linear feito, tem como único objetivo mostrar a tendência de comportamento dos pontos experimentais.

\section{Degradação em função da configuração de rosca}

A Figura 4 mostra os espectros de infravermelho do polipropileno nas regiões de interesse, para as amostras extrudadas 3 e 5 vezes, em todas as configurações de roscas, comparados ao polímero virgem. Nota-se o aumento da concentração de carbonilas e insaturações, $\left(1725 \mathrm{~cm}^{-1} \mathrm{e} 1650 \mathrm{~cm}^{-1}\right.$, respectivamente), sendo que as roscas de configurações $\mathrm{C} 90$ e C90E são as mais ativas na geração de produtos da degradação oxidativa do PP.

$\mathrm{Na}$ Figura 5 estão mostrados os resultados dos índices de carbonila (IC) e insaturações (IIns), em função da configuração de rosca para o polipropileno extrudado 3 e 5 vezes. Para cada amostra foram fei-
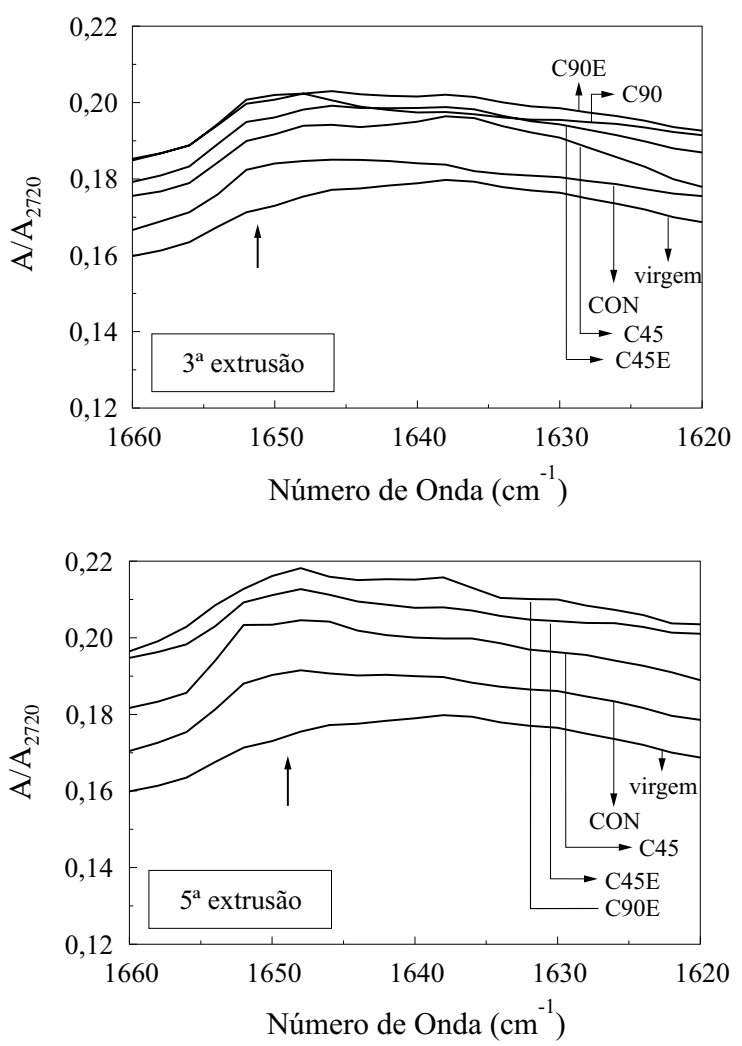

Figura 4. Aumento da concentração dos produtos da degradação (carbonilas e insaturações) em função da configuração da rosca e número de extrusões. 

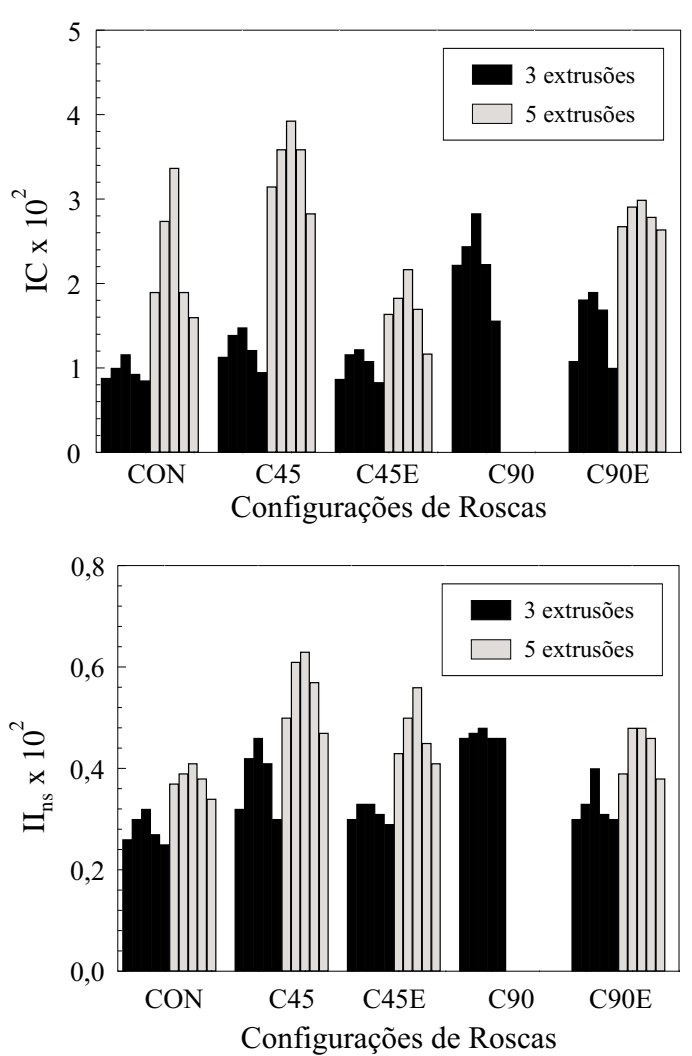

Figura 5. Índices de carbonila e insaturação em função da configuração da rosca com 3 e 5 extrusões.

tos cinco filmes para avaliação da reprodutibilidade dos dados. Em todas as configurações, tanto o índice de carbonila como o índice de insaturação aumentam com o número de extrusões. Os dois índices evidenciaram também, que a rosca de configuração C90, é a que realmente causa maior nível de degradação mecano-termoxidativa, mesmo quando comparada à rosca C90E. Isto era esperado para os casos de ele- mentos com nível de cisalhamento mais baixos (EM45) mas não no caso C90E. Tal fato será discutido adiante. Dados referentes à quinta extrusão do polipropileno com a rosca de configuração C90 não foram obtidos, pois foi impossível processar o polipropileno pela quinta vez nesta configuração.

A Tabela 2 apresenta os valores de pesos moleculares médios $\overline{\mathrm{M}}$ e $\overline{\mathrm{M}} \mathrm{w}$, e polidispersão $\frac{\overline{\mathrm{M}} \mathrm{w}}{\overline{\mathrm{n}}}$ para as amostras de polipropileno extrudadas 3 e 5 vezes em todas as configurações, assim como para o polipropileno como recebido, definido aqui como virgem.

\section{Degradação em função do número de extrusões}

A Figura 6 apresenta as curvas de distribuição de peso molecular, mostrando o efeito do número de extrusões. As curvas de distribuição de peso molecular referentes à quinta extrusão apresentam-se mais estreitas e deslocadas para a região de pesos moleculares menores, reduzindo principalmente as frações de maiores pesos moleculares.

\section{Degradação em função da configuração da rosca}

A Figura 7 mostra o efeito do tipo de elementos de mistura EM45 e EM90 nas curvas de distribuição de peso molecular para um mesmo número de extrusões. A degradação se processa com um estreitamento nas curvas de distribuição de peso molecular e deslocamento de toda a curva no sentido de pesos moleculares menores. Isto ocorre principalmente nas frações de altos pesos moleculares do material processado com roscas de configurações com elementos de mistura de 90 graus.

Tabela 2. Pesos moleculares e polidispersões de PP após múltiplas extrusões.

\begin{tabular}{ccccc}
\hline Extrusão & Configuração & $\overline{\mathbf{M}} \mathbf{n}$ & $\overline{\mathbf{M}} \mathbf{w} \mathbf{\overline { \mathbf { M } } \mathbf { w }}$ \\
\hline Virgem & - & 48.700 & 351.200 & 7,2 \\
\hline CON & 75.200 & 265.000 & 3,5 \\
3 & C45 & 41.500 & 203.000 & 4,9 \\
& C45E & 66.100 & 256.200 & 3,9 \\
& C90 & 64.100 & 161.800 & 2,5 \\
& C90E & 44.900 & 208.000 & 4,6 \\
\hline 5 & CON & 37.800 & 183.000 & 4,8 \\
& C45 & 47.700 & 154.900 & 3,2 \\
& C45E & 62.800 & 187.500 & 3,0 \\
& C90E & 35.000 & 163.600 & 4,7 \\
\hline
\end{tabular}


Babetto, A. C.; Canevarolo, S. V. - Degradação de Polipropileno durante múltiplas extrusões
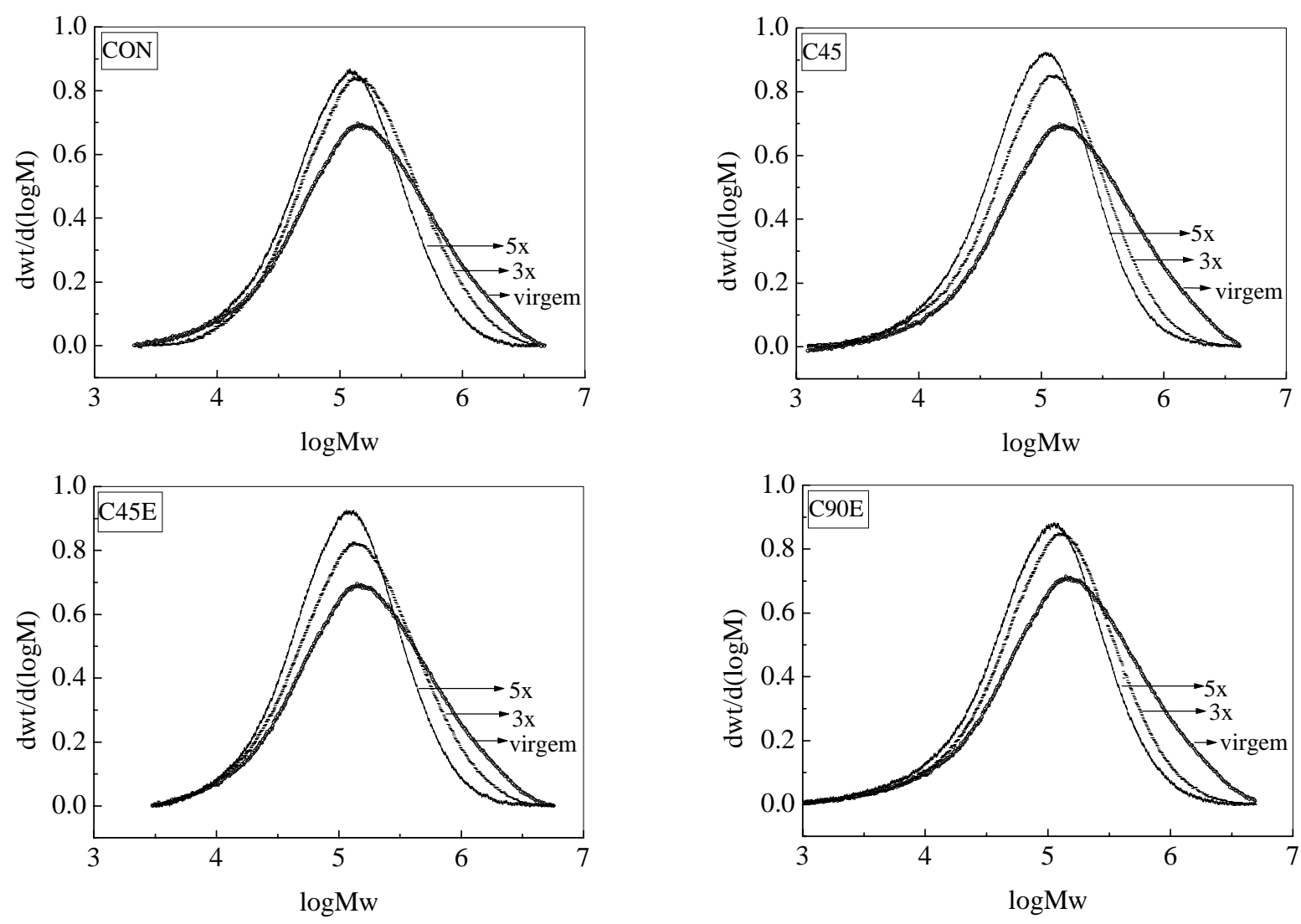

Figura 6. Distribuição de peso molecular após múltiplas extrusões para as configurações de rosca estudadas.
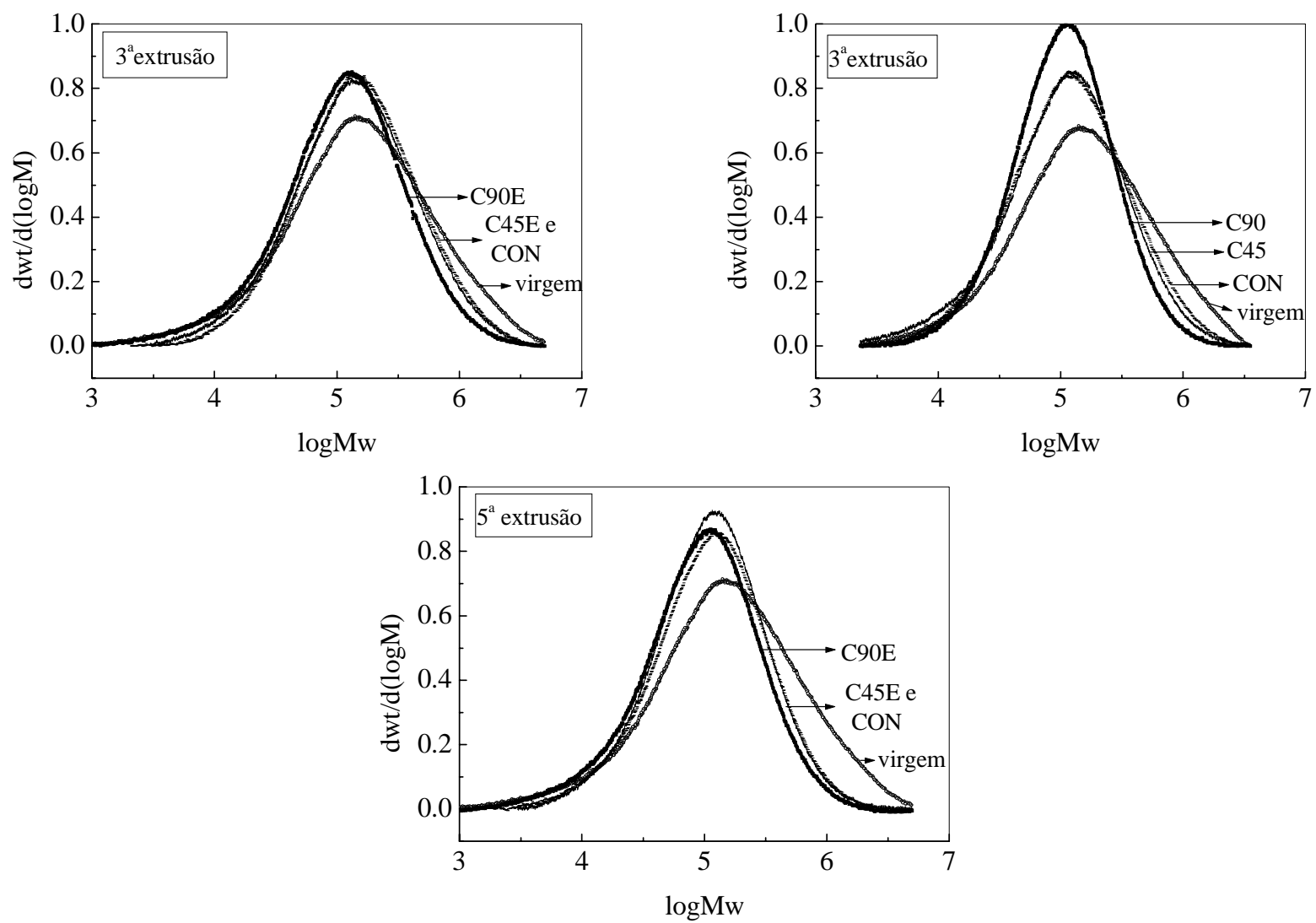

Figura 7. Efeito do tipo de elemento de rosca nas curvas de distribuição de peso molecular após múltiplas extrusões. 

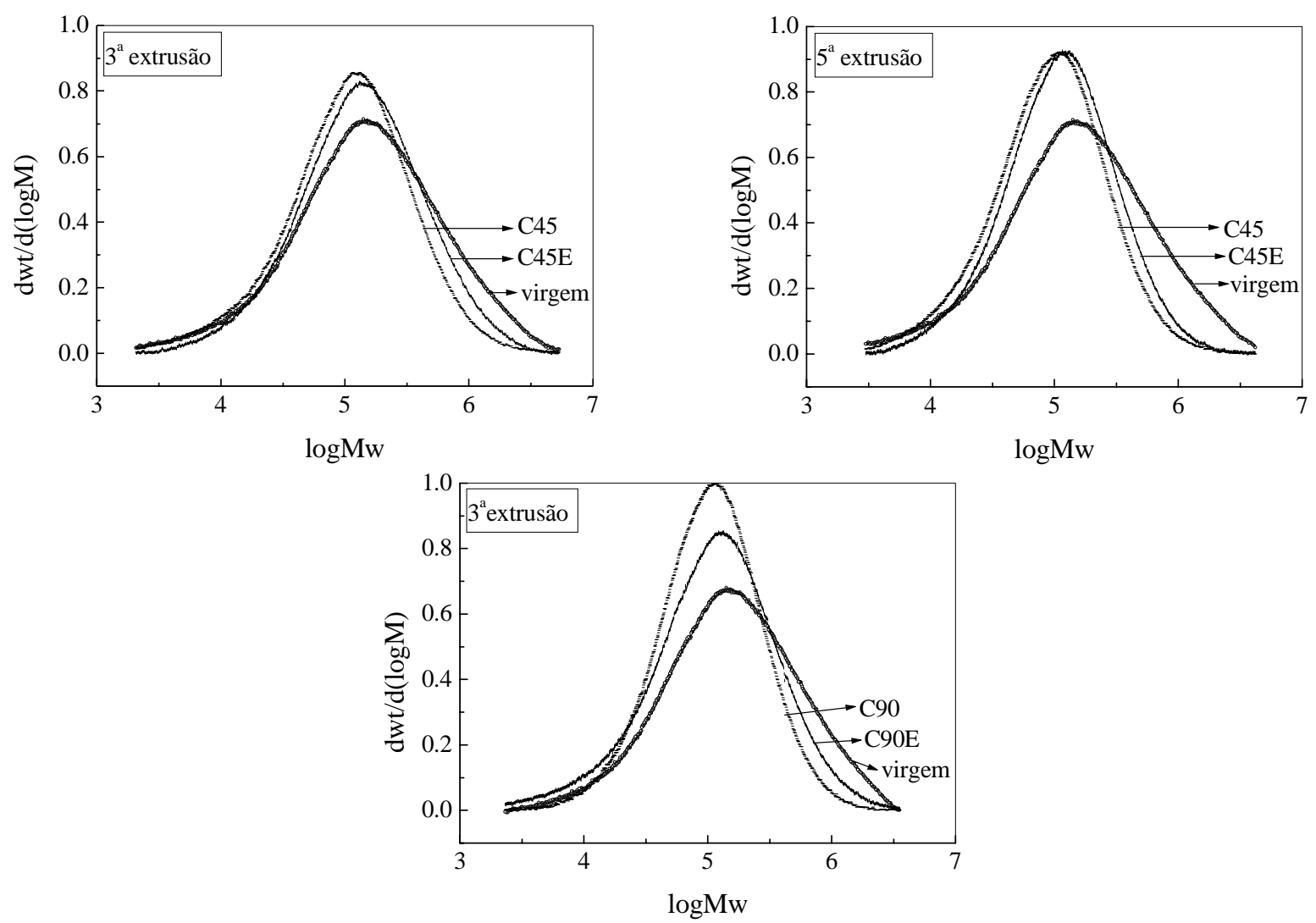

Figura 8. Efeito do elemento esquerdo nas distribuições de peso molecular de PP após múltiplas extrusões.

A Figura 8 apresenta as curvas de distribuição de peso molecular do polímero processado em configurações de rosca com mesmo tipo de elemento de mistura, diferindo pela presença de um elemento esquerdo ECE. Este elemento provoca menor deslocamento das curvas no sentido de baixos pesos moleculares. Este resultado é teoricamente inesperado ${ }^{[2,3]}$, pois o ECE gera contrafluxo fazendo o material retornar à região com os elementos de mistura, aumentando a ação cisalhante $\mathrm{e}$ portanto o nível de degradação mecânica. Porém, o comportamento das curvas de distribuição de peso molecular, assim como os índices IC e IIns indicam o contrário, ou seja, uma redução do grau de degradação.

Para explicar tal efeito calculou-se o volume de massa retida pelos elementos de mistura e de passo esquerdo. Este foi definido como sendo o volume de material fundido presente na extrusora com configuração de rosca cisalhante, que excede o volume de material fundido presente na extrusora com uma rosca de configuração composta apenas por elementos de condução (CON). A taxa de alimentação na extrusora é dada pela vazão mássica definida por:

$$
Q=\frac{\rho f^{-V}}{t}
$$

Onde: $Q$ : vazão mássica em $(\mathrm{g} / \mathrm{s}) ; \bar{t}$ : tempo de residência médio em $(\mathrm{s}) ; \rho_{f}$ : densidade do fundido em $\left(\mathrm{g} / \mathrm{m}^{3}\right) ; V$ : volume do fundido em $\left(\mathrm{m}^{3}\right)$.

Substituindo-se os valores de operação utilizados $\mathrm{Q}=5 \mathrm{~kg} / \mathrm{h}$ e $\rho_{f}=0,742 \mathrm{~g} / \mathrm{cm}^{3}$ (calculada segundo Zoller ${ }^{[18])}$, obtém-se a equação (2):

$$
V\left(\mathrm{~mm}^{3}\right)=1871,8\left(\mathrm{~mm}^{3} / \mathrm{s}\right) t(\mathrm{~s})
$$

De posse do valor do tempo de residência médio $(\bar{t})$ obtido segundo a metodologia de medidas de tempo de residência on-line ${ }^{[13,14]}$, a equação (2) permite o cálculo do volume de massa fundida contida na rosca da extrusora durante o processamento. $\mathrm{O}$ volume retido $\left(\mathrm{V}_{\mathrm{R}}\right)$ de fundido em uma dada rosca de configuração $\mathrm{C}$ pode ser obtido subtraindo-se o volume dessa configuração $\left(\mathrm{V}_{\mathrm{C}}\right)$ do volume de material retido na rosca de configuração de condução $\left(\mathrm{V}_{\mathrm{CON}}\right)$. Esta última é a configuração que proporciona menor quantidade de material retido na rosca, dada a sua alta capacidade de transporte. O cálculo do tempo de residência médio é função não só do tempo de residência inicial mas também do final. Como este último é de difícil definição e aqui se trata da diferença 
Babetto, A. C.; Canevarolo, S. V. - Degradação de Polipropileno durante múltiplas extrusões

Tabela 3. Tempos de residência iniciais ( $t$ ), volumes retidos $\left(V_{R}\right)$ e grau de preenchimento

\begin{tabular}{cccc}
\hline Configuração & $\begin{array}{c}\text { t } \\
(\mathbf{s})\end{array}$ & $\begin{array}{c}\text { VR } \\
\left(\mathbf{m m}^{\mathbf{3}}\right)\end{array}$ & $\begin{array}{c}\text { Grau de preenchimento } \\
(\boldsymbol{\%})\end{array}$ \\
\hline CON & 37,2 & - & 14,2 \\
C45 & 52,4 & 28452 & 19,7 \\
C45E & 57,2 & 37361 & 21,4 \\
C90 & 72,4 & 65888 & 27,4 \\
C90E & 75,7 & 72065 & 28,4 \\
\hline
\end{tabular}

entre dois tempos de residência optou-se pela substituição do tempo médio pelo tempo inicial que é de fácil definição e portando mais sensível. Com esta aproximação e para as condições de processamento usadas neste artigo, a equação para o cálculo de volume retido se torna:

$$
V_{R}\left(\mathrm{~mm}^{3}\right)=1871,82 \cdot\left(t_{\mathrm{C}}-t_{\mathrm{CON}}\right)
$$

onde $t_{C}$ e $t_{C O N}$ são os tempos de residência iniciais do material processados com roscas a uma dada configuração $(\mathrm{C})$ e de condução $(\mathrm{CON})$, respectivamente. Os valores de tempos de residências iniciais e volume retido para as configurações utilizadas neste estudo estão apresentados na Tabela 3.

As configurações de roscas com elementos de mistura EM90 retém o maior volume de polímero fundido em comparação às demais. Da mesma forma as configurações com elementos de passo esquerdo ECE retém também um volume maior de fundido que as configurações sem este elemento. Esses dados estão de acordo com a teoria ${ }^{[2]}$ sobre elementos de rosca que afirma que elementos EM90 não possuem capacidade de transporte e que os elementos EM45 possuem uma pequena capacidade. A retenção de uma maior quantidade de material fundido causa maior ocupação de volume no canal e portando reduz a quantidade disponível de oxigênio que seria utilizado para a oxidação do polímero fundido. Tal redução na disponibilidade de oxigênio reduz o nível de oxidação do polímero. Também foi calculado o grau de preenchimento ${ }^{[1]}$ do canal da rosca, definido como a razão entre o volume de polímero no canal da rosca e o volume do canal vazio. O volume do canal da rosca foi calculado, respeitando-se a geometria de cada tipo de elemento de rosca, e os volumes de polímero no canal através da equação (3). Os graus de preenchimento para as roscas de cada configuração estão apresentados na
Tabela 3, onde as roscas de configuração com ECE, possuem valores maiores em relação às configuração similares sem estes elementos, concordando com os valores de volume retido. Apesar das configurações C90 e C90E possuírem maior volume retido no canal da rosca, maior é o nível de degradação do material em relação às configurações C45 e C45E, pois a agressividade mecânica é maior. Para simplificar o cálculo do grau de preenchimento assumiuse que este é uniforme axialmente, o que não é sempre verdade, principalmente nas configurações com zona cisalhante. Os valores de grau de preenchimento para todas as configurações são baixos, dadas as condições operacionais impostas na extrusora que foram de alta rotação e baixa taxa de alimentação.

\section{Conclusão}

O aumento do número de extrusões e agressividade da configuração da rosca causa no polipropileno o decréscimo do peso molecular e estreitamento de sua distribuição, assim como, o aumento da concentração de carbonilas e insaturações no polímero. A degradação é por cisão de cadeias de maiores pesos moleculares aumentando a proporção das de baixo peso molecular. Elementos de mistura com ângulos de 90 graus (EM90) produzem maior nível de degradação que elementos de mistura de 45 graus (EM45) e elementos de condução (EC). A adição de elementos esquerdos (ECE) produzem menor nível de oxidação pois aumentam o volume da massa retida, reduzindo a disponibilidade de oxigênio dentro do barril necessário para a oxidação do fundido.

\section{Agradecimentos}

Os autores agradecem ao suporte financeiro da CAPES, FAPESP (projeto temático 96/8550-2) e CNPq (projeto PRONEX 41.96.0872.00). 


\section{Referências Bibliográficas}

1. Rauwendaal, C. Polymer Extrusion. Hanser Publishers, Munich, (1986).

2. Dreiblatt A., Eise, K., Mixing in Polymer Processing, cap. 5.

3. Eise, K.; Herrman, H.; Jakopin, S.; Burkhardt, U.; Werner, H., Advan. Plast. Technol. 1, 18 - 39, (1981).

4. Ebner, K. , White, J. L.,Intern. Polym. Proces. IX, 3, (1994).

5. Cheng, H., Manas-Zloczower, I. , Polym. Eng. Sci., Julho, (1997), vol. 37, n 6.

6. White, J. L., Chen, Z. Polym. Eng. Sci., Fev., 1994,vol. $34, \mathrm{n} 3$.

7. Stade, K. H., Kunststoffe German Plastics, 73 (1983) 6.

8. Reich, L, Stivala,S. S., Elements of Polymer Degradation, McGraw-Hill, (1971).

9. Tzoganakis, J., Vlachopoulos, Hamielec, A. E., Polym. Eng. Sci., Março,(1989), vol 29, n.6.
10. Kim B. J. , White, J. L. Intern. Polym. Proces. X, 3, (1995).

11. Hinsken H., S. Moss, S., Pauquet J., Zweifel, H., Polym. Degrad. Stab., (1991), vol. 34.

12. González-González, V. A., Neira-Velázquez, G., Angulo-Sánchez, J. L., Polym. Degrad. Stab., vol 60, (1998), 33-42.

13. Santos, D. M., Canevarolo, S. V., XIII CBECIMAT, 4166 - 4175, 6-9/Dez/1998, Curitiba/ PR.

14. Santos, D. M., Canevarolo, S. V., Polim. Cien. Tec. ABPol, n 2, Abr/Jun, 60-69 (1999).

15. Rabello, M. S., White, J.R., ., Polym. Degrad. Stab., vol 56, (1997), 55-73.

16. Garton, A.,Carlsson, D.J., Wiles, D. M., J. Polym. Sci. Polym. Chem. Ed, Vol. 16, 33-40 (1978).

17. Tobin, M. C., J. Phys. Chem., Vol. 64, 216 - 219 (1960).

18. Zoller, P., J. Polym. Sci., Vol. 23, 1057-1061 (1979).

Recebido:23/08/99

Aprovado:24/05/00 